\title{
Efficient Surveillance of Childhood Diabetes Using Electronic Health Record Data
}

\author{
Victor W. Zhong ${ }^{\star 1}$, Jihad S. Obeid ${ }^{2}$, Jean B. Craig' ${ }^{2}$, Emily R. Pfaff ${ }^{1}$, Joan Thomas', \\ Lindsay M. Jaacks ${ }^{3}$, Daniel P. Beavers ${ }^{4}$, Timothy S. Carey ${ }^{1}$, Jean M. Lawrence ${ }^{5}$, Dana \\ Dabelea $^{6}$, Richard F. Hamman ${ }^{6}$, Deborah A. Bowlby ${ }^{2}$, Catherine Pihoker ${ }^{7}$, Sharon H. \\ Saydah $^{8}$ and Elizabeth J. Mayer-Davis ${ }^{1}$
}

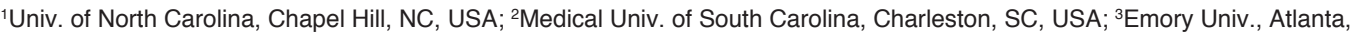
GA, USA; ' Wake Forest Univ., Winston-Salem, NC, USA; ${ }^{4}$ Kaiser Permanente Southern California, Pasadena, CA, USA; ${ }^{6}$ Univ. of Colorado, Denver, CO, USA; ${ }^{7}$ Univ. of Washington, Seattle, WA, USA; ${ }^{8} \mathrm{CDC}$, Atlanta, GA, USA

\section{Objective}

The study goal was to develop an efficient surveillance approach for childhood diabetes across two large Southeastern US public academic health care systems, using electronic health record (EHR) data.

\section{Introduction}

Traditional surveillance methods, such as registries that require manual validation of every diabetes case or questionnaires, are resource intensive and associated with considerable delay in reporting results. An EHR-based surveillance system may be more efficient for sustained monitoring of the incidence and prevalence of childhood diabetes, so as to inform health care needs for this growing population.

\section{Methods}

The study population at the Medical University of South Carolina (MUSC) included all children $<20$ years of age as of December 31,2012 who were seen by a health care provider for any reason between July 1, 2012 and December 31, 2012. At the University of North Carolina Health Care System (UNC-HCS), we included all children $<20$ years of age as of December 31, 2011 who were seen by a health care provider for any reason in 2011. EHR data included demographics, billing codes, outpatient medications prescription lists, laboratory test results and patient problem lists. Presumptive diabetes cases were identified as those having $\geq 1$ of the following 5 indicators in the past 3.5 years, including elevated $\mathrm{HbA} 1 \mathrm{c}$, elevated blood glucose, diabetes related billing codes, diabetes related patient problem lists or outpatient medications. EHRs of the presumptive cases were manually reviewed, and true diabetes status and diabetes type were determined by the presence of a diabetes diagnosis in the EHRs written by health care providers. Algorithms for identifying diabetes cases overall and classifying type were either pre-specified or derived from classification and regression tree analysis. Surveillance approach was developed based on the best algorithms identified.

\section{Results}

We used pre-specified algorithms derived from billing codes only and targeted manual EHRs review to develop a stepwise surveillance approach (Figure 1A and 1B). The sensitivity and positive predictive value for this surveillance approach in both health care systems were generally $\geq 90 \%$ for ascertaining diabetes cases overall, and classifying cases with type 1 or type 2 diabetes (Table 1). This stepwise surveillance approach resulted in a $>70 \%$ reduction in the number of cases requiring manual validation compared to traditional surveillance methods.

\section{Conclusions}

EHR data may be used to establish an efficient and accurate approach for large scale surveillance for childhood diabetes, although some manual effort is still needed.
Table 1. The Performance of the Stepwise Surveillance Approach

\begin{tabular}{|c|c|c|c|c|}
\hline & \multicolumn{2}{|c|}{$\begin{array}{c}\text { MUSC review 289 (27.4\%) of 1,055 } \\
\text { records }\end{array}$} & \multicolumn{2}{|c|}{ UNC-HCS review 234 (18.2\%) of 1,289 } \\
& Sensitivity, \% & $\begin{array}{c}\text { Positive predictive value, } \\
\%\end{array}$ & Sensitivity, \% & Positive predictive value, \% \\
\hline $\begin{array}{c}\text { Total diabetes } \\
\text { cases }\end{array}$ & 97.9 & 96.7 & 97.0 & 96.8 \\
\hline $\begin{array}{c}\text { Type 1 diabetes } \\
\text { cases }\end{array}$ & 99.6 & 93.9 & 99.3 & 94.8 \\
\hline $\begin{array}{c}\text { Type 2 diabetes } \\
\text { cases }\end{array}$ & 91.5 & 100.0 & 89.5 & 100.0 \\
\hline $\begin{array}{c}\text { Other diabetes } \\
\text { cases* }\end{array}$ & 79.2 & 100.0 & 80.4 & 100.0 \\
\hline
\end{tabular}

* Other diabetes cases included diabetes cases that were neither type 1 nor type 2

Figure 1A. The stepwise surveillance approach at MUSC

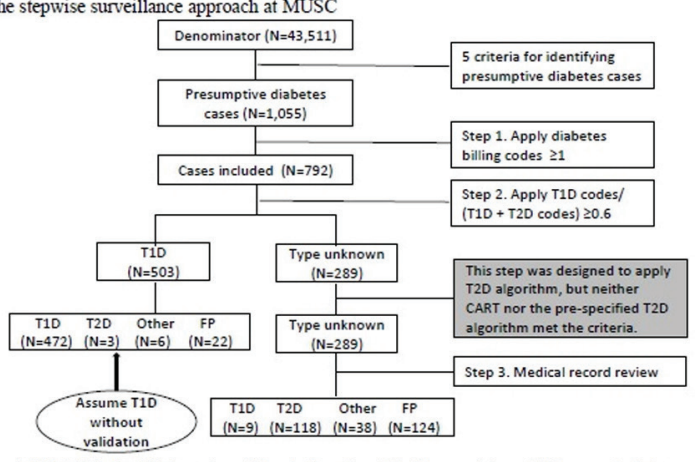

Abbreviations: MUSC, Medical University of South Carolina; FP, false positives; T1D, type 1 diabetes T2D, type 2 diabetes.

Figure 1B. The stepwise surveillance approach at UNC Health Care System

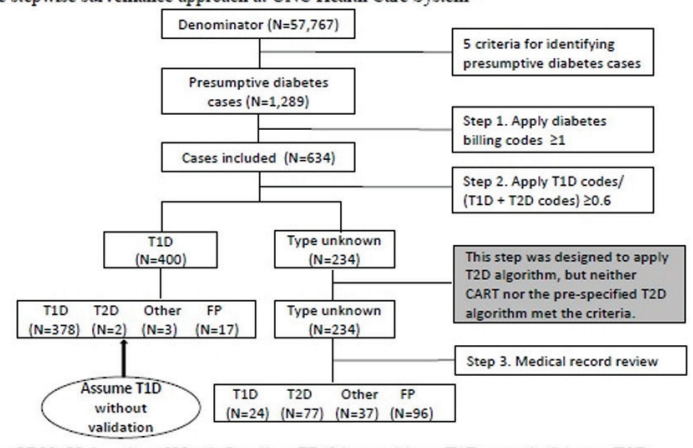

Abbreviations: UNC, University of North Carolina; FP, false positives; T1D, type 1 diabetes; T2D, type 2 diabetes.

\section{Keywords}

Childhood diabetes; Surveillance; Electronic health record 


\section{Acknowledgments}

This study is funded by the CDC (PA numbers 00097, DP-05-069, and DP-10-001) and supported by the National Institute of Diabetes and Digestive and Kidney Diseases and Sanofi Global Scholars Program.

\section{References}

Zhong VW, Pfaff ER, Beavers DP, et al. Use of administrative and electronic health record data for development of automated algorithms for childhood diabetes case ascertainment and type classification: the SEARCH for Diabetes in Youth Study. Pediatr Diabetes. 2014;15(8):573-84.

\section{*Victor W. Zhong}

E-mail: wzhong@live.unc.edu 